\title{
Calculation of measurement uncertainty for cellulose based transformed board material in tensile testing
}

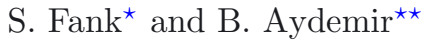 \\ TÜBITAK UME, Force Group Laboratory, Gebze, Kocaeli, Turkey
}

Received: 16 March 2012 / Accepted: 24 March 2012

\begin{abstract}
Today's global economy obliges manufacturers to produce their products in high quality with cheaper cost. Thus, manufacturers must develop their products applying tests to get better performance and higher quality before distributing them to the competitive market. In order to produce long working life of transformer reactors without losing their performance, different tests including axial and flexural testing are applied on the cellulose based laminated board material used in production. During development of laminated board material, test results should be known very well and the measurement uncertainty of tensile tests should be calculated taking into account all parameters. In this study, effective parameters on the quality of tensile testing causing an increase on the measurement uncertainty are investigated. Tensile strength and elongation test of laminated board material were carried out according to IEC 60641-2 and the uncertainty contributions based on experimental results were calculated. The results and impact of the uncertainty parameters are presented in detail.
\end{abstract}

Keywords: Tensile testing; measurement uncertainty; laminated pressboard

\section{Introduction}

The material supply and related industries are worth trillions US $\$$ each year over the world. In all cases, the measurement of the material properties by mechanical testing is very important for using and developing new materials.

The quality of the mechanical parts such as bolts, machines elements, aircraft parts etc. is controlled by testing the material properties (such as the tensile strength, lower yield stress, proof stress, impact strength, Brinell, Rockwell and surface hardness, elongation after fracture). Each of these properties is measured according to an appropriate test method for tensile properties $[1,2]$.

Several tests are necessary during the development process of the products. The tensile testing is applied on the materials used in research studies, in civil engineering, military applications, materials development, automotive, ship and aircraft industry. In order to make precise and accurate tensile testing, all influencing parameters on the test results must be analyzed very well. In this way, such parameters can be controlled and lower measurement uncertainty can be achieved in order to make true conformity assessment for defected parts resulting in less scrap.

For the insulation of coil sheet of transformers, cellulose based laminated pressboard material are used. Mechanical material properties of the laminated pressboard are usually obtained through a series of experiments, fol-

\footnotetext{
${ }^{\star}$ Correspondence: sinan.fank@ume.tubitak.gov.tr

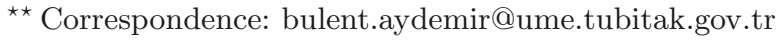

lowing appropriate standards, such as IEC 60641-2 (2004) and IEC 60763-2 (2007) [3,4]. However, the available standards for laminated pressboard materials do not provide an uncertainty budget and do not present the calculation of measurement uncertainty.

This study explains the calculations of measurement uncertainty for tensile testing of laminated pressboard materials. The calculation model can also be used for tensile testing of all other materials.

\section{Laminated pressboard material}

Pressboard is cellulose based laminated material and is manufactured with hot-press-dried method. It is made from highly purified unbleached softwood pulp from Russia and Canada. The resins in use are casein and polyester glues; casein is aqueous, polyester is nonaqueous. It has obvious good properties in mechanical strength, electrical strength and compressibility is used worldwide as insulation material for transformers reactor and bushing current transformer in the electric transmittal field. Typical applications of laminated pressboard are pressure rings, static rings, beams, clamping plates and support strips, etc.

The conditioning shall be made at $23 \pm 2{ }^{\circ} \mathrm{C}$ and $(50 \pm 5) \%$ relative humidity until the moisture content of the specimen reaches $5.5 \%$ to $8 \%$. The conditioning shall be approached from the dry side after drying at $70 \pm 5{ }^{\circ} \mathrm{C}$ 


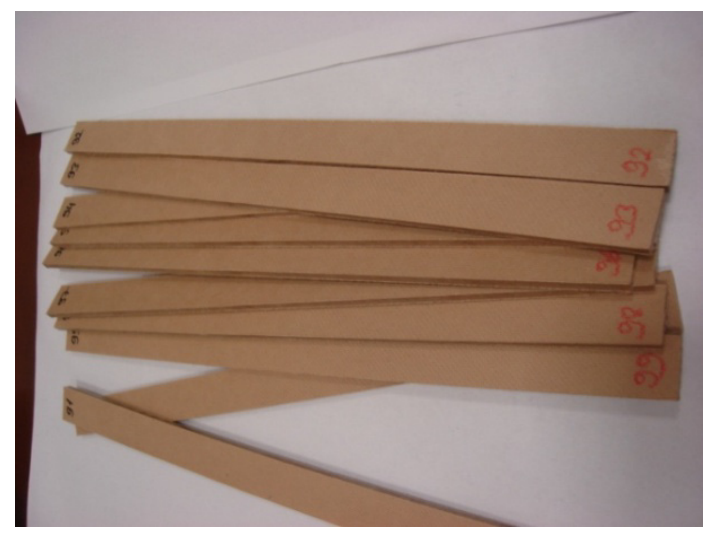

Fig. 1. (Color online) Laminated pressboard test bars.

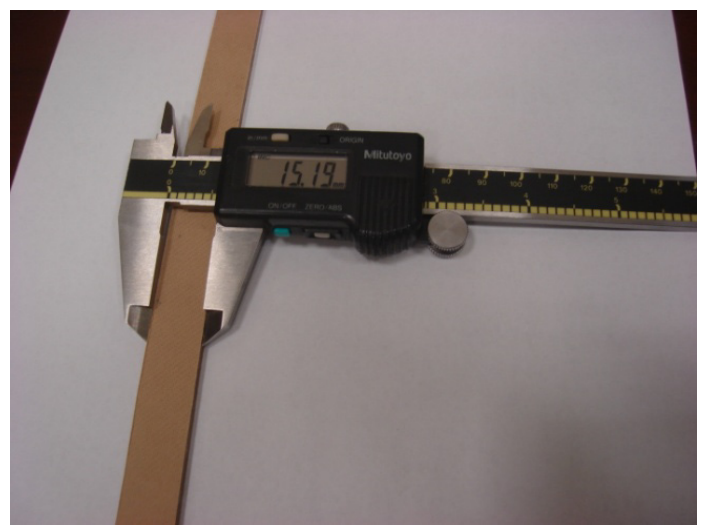

Fig. 2. (Color online) Measurement of test bar dimensions by caliper.

for a period sufficient to ensure that the conditioning atmosphere produces a mass increase in the specimen [3].

\section{Uncertainty analysis}

Material testing machines are calibrated according to EN ISO 7500-1 for requirements of its force measuring system [5]. The calibration certificates of the testing machine mentions only that they meet EN ISO 7500-1 standard requirements for force measuring system of the testing machine. This standard requires that the indication of the testing machine has to be correct to within specified limits given in those standards [5].

In order to determine combined uncertainty of test results of laminated board or any materials first take into account the calibration uncertainties of the testing machine used to measurement of tensile force and micrometer used for measurement of dimensions of tested specimens. Then, the standard deviation of the uncertainty of a tensile strength measurement is calculated in the 5 test results.

Sensitivity coefficients are essentially conversion factors that allow one to convert the units of an input quantity into the units of the measured [2]. Sensitivity coefficients measure of how much change is produced in the

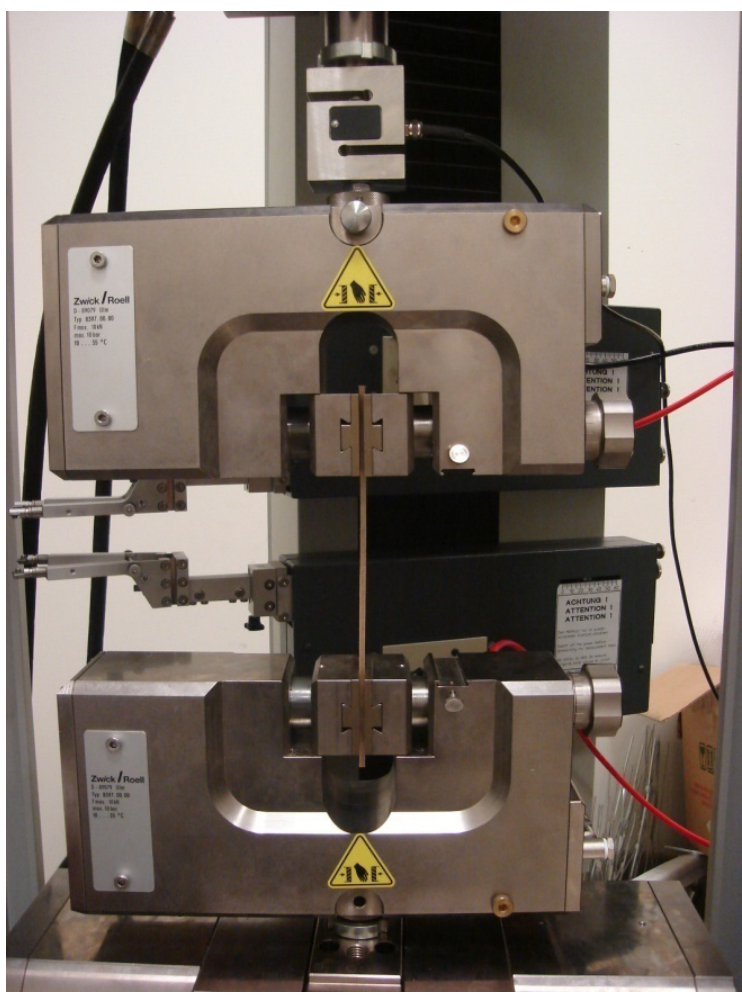

Fig. 3. (Color online) Measurement of test bars during tensile testing.

measured by changes in an input quantity. Mathematically, sensitivity coefficients are obtained from partial derivatives of the model function $f$ with respect to the input quantities. In particular, the sensitivity coefficient $c_{i}$ of the input quantity $x_{i}$ is given by which expresses mathematically how much $f$ changes given an infinitesimal change in $x_{i}[2]$.

$$
c_{i}=\frac{\partial f}{\partial c_{i}}
$$

In this case, it can be determined the model function of the tensile strength to find the measurement uncertainty of tensile strength as below;

$$
\sigma=\frac{F}{A}
$$

where $\sigma$ is the tensile strength, $F$ is the maximum force and $A$ is the cross-section of the tested bar. The sensitivity coefficients, $c_{F}$ and $c_{A}$ can be obtained easily as follows:

$$
\begin{aligned}
& c_{F}=\frac{\partial \sigma}{\partial F}=\frac{1}{A} \\
& c_{A}=\frac{\partial \sigma}{\partial A}=-\frac{F}{A^{2}} .
\end{aligned}
$$

Once, all values of the uncertainty contributor $u_{i}$ have been estimated and reduced to one standard deviation, and the sensitivity coefficients $c_{i}$ have been determined. It is usually necessary only to "root-sum-square" their products, i.e., take the square root of the sum of the squares 
of the uncertainty estimates multiplied by the squares of their corresponding sensitivity coefficients, in order to determine combined standard uncertainty $u_{c},[2]$.

$$
u_{c}=\sqrt{\sum_{i} c_{i}^{2} \times u_{i}^{2}}
$$

Measurement uncertainty for tensile strength $\left(u_{\sigma}\right)$ can be written as follows [8],

$$
u_{\sigma}=\sqrt{c_{F}^{2} \times u_{F}^{2}+c_{A}^{2} \times u_{A}^{2}}
$$

$c_{F}$ : sensitivity coefficient for applied force measurement;

$c_{A}$ : sensitivity coefficient for cross-section measurement of test bar;

$u_{F}$ : measurement uncertainty of applied force taken directly from calibration certificate of material testing machine;

$u_{A}$ : measurement uncertainty of cross-section of the test bar.

Cross-section of the test bar is calculated as in equation (7),

$$
A=a \times b
$$

$A$ : cross-section of the test bar;

$a$ : thickness of the test bar;

$b$ : width of the test bar.

In order to calculate measurement uncertainty of crosssection of the test bar $\left(u_{A}\right)$ can be calculated as below;

$$
u_{A}=\sqrt{c_{A_{c}}^{2} \times u_{A_{c}}^{2}}
$$

$c_{A c}$ : sensitivity coefficient for cross-section measurement of test bar;

$u_{A c}$ : measurement uncertainty of cross-section of the test bar.

Using with equation (7), sensitivity coefficient for crosssection measurement of test bar $\left(c_{A c}\right)$ can be calculated as in equation (9);

$$
c_{A_{c}}=a+b .
$$

Calculation of measurement uncertainty $\left(u_{A c}\right)$ of crosssection of the test bar is depending on the measurement uncertainty of caliper (or micrometer) taken from calibration certificate of caliper $\left(U_{c-c e r t}\right)$, and depending on standard deviation of the successive measurement (at least five) of the test bar with caliper can be called standard uncertainty of measurement taken with caliper $\left(u_{c-s t}\right)$.

$$
u_{A_{c}}=\sqrt{\left(\frac{U_{c-c e r t}}{2}\right)^{2}+u_{c-s t}^{2}}
$$

$\left(\mathrm{U}_{\text {c-cert }}\right)$ is divided by 2 due to the calibration certificate prepared according to the coverage factor $k=2$ for $95 \%$ confidence level.

Standard uncertainty of the measurements taken from caliper $\left(u_{c-s t}\right)$ is calculated as taking standard deviation of the successive measurement (at least five) of the test bar given for both thickness $(a)$ and width $(b)$ given in below;

$$
\begin{aligned}
u_{c-s t, a} & =\frac{s_{c, a}}{\sqrt{n_{a}}} \\
u_{c-s t, b} & =\frac{s_{c, b}}{\sqrt{n_{b}}} \\
u_{c-s t} & =\sqrt{u_{c, a}^{2} \times u_{c, b}^{2}}
\end{aligned}
$$

$S_{c, a}, S_{c, b}$ : standard deviation of the successive measurement taken by caliper for both thickness $(a)$ and width $(b)$;

$n_{a}, n_{b}$ : number of measurement for thickness $(a)$ and width $(b)$;

$u_{c^{-} s t}$ : combined standard uncertainty of thickness $(a)$ and width (b) measurements by caliper

$$
s_{c}=\sqrt{\frac{\sum_{i=1}^{n}\left(X_{i}-\bar{X}\right)^{2}}{n-1}}
$$

$X_{i}$ : value of measurement;

$\bar{X}$ : average of the measurements.

Estimation of measurement uncertainty of tested bar for specified materials depends on several error sources such as $[6-8]$ :

- measurement of test bar dimensions (already added into the calculations);

- measurement of tensile force (already added into the calculations);

- test temperature and loading rates in successive stages of tests;

- the method of gripping the test piece and axiality of the application of the force;

- the testing machine characteristics (stiffness, drive, control and method of operation);

- human and software errors associated with determination of the tensile properties;

- the material in homogeneity that exists even within a single processed batch obtained from a single melt of material.

The first two error sources that are measurement of test bar dimensions and measurement of tensile force have already been added into the measurement uncertainty calculations. The calculations of other effects are very difficult due to the determination of all influencing parameters. Instead of uncertainty calculations for all listed error sources, the standard deviation of the test results can be used to calculate the measurement uncertainty of all other parameters mentioned in the listed error sources. The test data covers the influencing parameters. The standard deviation of test data gives the standard uncertainty of testing $\left(u_{\text {test }}\right)$ as a combined uncertainty of the above error sources;

$$
u_{\text {test }}=\frac{s_{\text {test }}}{\sqrt{n_{\text {test }}}}
$$


$S_{\text {test }}$ : standard deviation of the test results of five test bars for tensile strength;

$n_{\text {test }}$ : number of test results for tested bars.

$$
s_{\text {test }}=\sqrt{\frac{\sum_{i=1}^{n}\left(X_{i}-\bar{X}_{\text {test }}\right)^{2}}{n_{\text {test }}-1}}
$$

$X_{i}$ : value of the tensile strength measurement;

$\bar{X}_{\text {test }}$ : average of the tensile strength measurement;

Then, combined uncertainty $\left(U_{\text {combined }}\right)$ can be calculated the measurement uncertainty for tensile strength $\left(u_{\sigma}\right)$ and test uncertainty $\left(u_{\text {test }}\right)$ as shown below;

$$
u_{\text {combined }}=\sqrt{u_{\sigma}^{2}+u_{\text {test }}^{2}} .
$$

Estimated expanded uncertainty $\left(U_{\text {exp }}\right)$ can be calculated as;

$$
U_{\text {exp }}=k \times u_{\text {combined }} \text {. }
$$

\section{Sample calculations}

The test result of the five test bars tested in material testing machine are given in Table 1 . The calculation of measurement uncertainties of the test results using equations from (3) to (18) are given in Table 2.

$\left(u_{F}\right)$ is directly taken from calibration certificate of material testing machine and $\left(U_{c \text {-cert }}\right)$ is also taken from the calibration certificate of the caliper.

The average thickness of the test bars is $20.13 \mathrm{~mm}$, the average width is $15.17 \mathrm{~mm}$, the average maximum force is $32163.07 \mathrm{~N}$ and the average tensile strength is $105.27 \mathrm{~N} / \mathrm{mm}^{2}$. The tensile strength and uncertainty in the result of tensile test are shown in Tables 1 and 2 as $105.27 \pm 2.32 \mathrm{~N} / \mathrm{mm}^{2}$.

Sample calculation shows that all of the uncertainty sources including dimensions of tested bars, type of crosssection, uncertainty of calipers, applied forces, repeatability of test results and related sensitivity coefficients can be founded step by step and added to the test results. When the test results are given in the report including uncertainty value, it can be more preferable and more reliable for the customer.

\section{Conclusions}

Analysis of the uncertainty sources incorporated during measurements of the tensile strength of the laminated pressboard material has been performed. All sources of uncertainty have been investigated in detail. They are dimension of tested bars, type of cross-section, uncertainty of calipers and applied forces and repeatability of test results. Examples illustrate the importance of uncertainty sources relevant to the variability of the parameters measured from series of tests bars in same sheet of laminated
Table 1. Tensile test results for laminated pressboard specimens.

\begin{tabular}{cccccc}
\hline $\begin{array}{c}\text { Test } \\
\text { bar }\end{array}$ & $a,(\mathrm{~mm})$ & $b,(\mathrm{~mm})$ & $\begin{array}{c}\text { Cross-section } \\
\text { of test bar } \\
A,\left(\mathrm{~mm}^{2}\right)\end{array}$ & $\begin{array}{c}\text { Tensile } \\
\text { force } \\
F,(\mathrm{~N})\end{array}$ & $\begin{array}{c}\text { Tensile } \\
\text { strength } \\
\sigma\left(\mathrm{N} / \mathrm{mm}^{2}\right)\end{array}$ \\
\hline 1 & 20.12 & 15.17 & 305.22 & 31924 & 105 \\
2 & 20.15 & 15.18 & 305.88 & 31928 & 104 \\
3 & 20.13 & 15.19 & 305.77 & 32000 & 105 \\
4 & 20.08 & 15.15 & 304.21 & 31403 & 103 \\
5 & 20.19 & 15.18 & 306.48 & 33560 & 109 \\
\hline$X$ & 20.13 & 15.17 & 305.51 & 32163.07 & 105.27 \\
\hline
\end{tabular}

Table 2. Calculations of sensitivity coefficients and uncertainties of the tensile testing.

\begin{tabular}{ccccc}
\hline $\begin{array}{c}C_{F}, \\
\left(1 / \mathrm{mm}^{2}\right)\end{array}$ & $\begin{array}{c}C_{A}, \\
\left(\mathrm{~N}^{2} / \mathrm{mm}^{4}\right)\end{array}$ & $\begin{array}{c}C_{A c}, \\
(\mathrm{~mm})\end{array}$ & $\begin{array}{c}u_{\sigma}, \\
\left(\mathrm{N} / \mathrm{mm}^{2}\right)\end{array}$ & $\begin{array}{c}U_{\text {exp }} \\
(\mathrm{mm})\end{array}$ \\
\cline { 1 - 4 } 0.000031 & -0.34 & 35.31 & 2.05 & \\
\cline { 1 - 4 }$u_{F}$ & $u_{A}$ & $u_{A c}$ & $u_{\text {test }}$ & \\
$0(\mathrm{~N})$ & $\left(\mathrm{mm}^{2}\right)$ & $\left(\mathrm{mm}^{2}\right)$ & $\left(\mathrm{N} / \mathrm{mm}^{2}\right)$ & \multirow{2}{*}{2.32} \\
\cline { 1 - 4 } 38.31 & 35.31 & 0.02 & 1.09 & \\
\hline
\end{tabular}

board material. The test results were reported with the calculated uncertainty values, and their impact is studied. It is expected that this will provide the transformer producers with selecting better material for safety and longer life of their end product as well as conducting further improvements. Besides, the given uncertainty calculation model in the measured properties following specific test procedures can be a guide for better testing procedures.

\section{References}

1. Evolving Needs for Metrology in Material Property Measurements, Report of the CIPM ad hoc Working Group on Materials Metrology (WGMM) (2007)

2. T.M. Adams, A2LA Guide for Estimation of Measurement Uncertainty in Testing, Guidance, G104 (2002)

3. IEC 60763-2, Specification for laminated pressboard Part 2: Methods of test, edition 2.0 (2007)

4. IEC 60641-2, Pressboard and presspaper for electrical purposes - Part 2: Methods of tests, 2nd edn. (2004)

5. EN ISO 7500-1, Metallic materials - Verification of static uniaxial testing machines, Part 1: Tension/compression testing machines-Verification and calibration of the forcemeasuring system (2004)

6. D.J. Lekou, T.T. Assimakopoulou, T.P. Philippidis, Estimation of the Uncertainty in Measurement of Composite Material Mechanical Properties During Static Testing, Strain 47, 430-438 (2011)

7. Room Temperature Tensile Testing: A method for Estimating Uncertainty of measurements, http://midas.npl. co.uk

8. B. Aydemir, S. Fank, Training notes for uncertainty calculation of mechanical tests (tensile and bending), G2KV-100, G2KV-100 (TÜBITAK UME, Turkey, 2011) 